\title{
Stability of the Endemic Coexistence Equilibrium for One Host and Two Parasites
}

\author{
T. Dhirasakdanon and H. R. Thieme* \\ School of Mathematical and Statistical Sciences \\ Arizona State University, Tempe, AZ 85287-1804, USA
}

\begin{abstract}
For an SI type endemic model with one host and two parasite strains, we study the stability of the endemic coexistence equilibrium, where the host and both parasite strains are present. Our model, which is a system of three ordinary differential equations, assumes complete crossprotection between the parasite strains and reduced fertility and increased mortality of infected hosts. It also assumes that one parasite strain is exclusively vertically transmitted and cannot persists just by itself. We give several sufficient conditions for the equilibrium to be locally asymptotically stable. One of them is that the horizontal transmission is of density-dependent (mass-action) type. If the horizontal transmission is of frequency-dependent (standard) type, we show that, under certain conditions, the equilibrium can be unstable and undamped oscillations can occur. We support and extend our analytical results by numerical simulations and by two-dimensional plots of stability regions for various pairs of parameters.
\end{abstract}

Key words: coexistence of parasite strains, disease incidence, SI endemic model, Routh-Hurwitz conditions, undamped oscillations, vertical transmission, horizontal transmission, disease-related fertility reduction.

AMS subject classification: 92D30, 34C25, 34D20

\section{Introduction}

There is a longstanding interest in the mechanisms that can cause undamped oscillations in endemic models: see $[2,10,14,15,16,18,19,22,23,37,39,40,43]$ and the references therein. One

\footnotetext{
*Corresponding author. E-mail: thieme@math.asu.edu
} 
class of mechanisms is the presence of multiple hosts and/or multiple parasites or multiple strains of one parasite [3, 4, 20, 24, 26, 27, 28, 44, 45].

This paper continues the investigation of an endemic model with one host and two strains of one parasite (or two different parasites) where one strain is exclusively vertically transmitted (the VT strain) and the other strain is horizontally (and possibly also vertically) transmitted (the HT strain) $[9,13]$. A similar scenario in a somewhat different model has been investigated in [1] (see Section 8 for a more detailed comparison).

Our model which consists of three ordinary differential equations has some remarkable properties. The first is the paradox that the ratio of horizontal to vertical transmission decreases if the coefficient, $\sigma$, of horizontal transmission increases [13]. This phenomenon has also been observed in the simulations of a discrete-time model [31]. The second is that the vertically transmitted strain that would go extinct by itself can persist by protecting the host against the more virulent horizontally transmitted strain [9]. (This had already been observed in simulations [29, 31].)

In this paper, we turn to the stability of the endemic coexistence equilibrium (ECE) (where the host and both parasite strains are present). Among other things, we will establish that the ECE is locally asymptotically stable for density-dependent (alias mass-action) incidence, but can lose its stability in an oscillatory fashion for frequency-dependent (alias standard) incidence. While a similar relation between incidence and stability has been observed for an HIV/AIDS model with infection-age dependent infectivity [35, 42], most of the time the relation has been found the other way round: stability for frequency-dependence incidence, but possible instability for densitydependent incidence $[16,24,37]$. Because our findings are different from what one may expect, we will present the application of the well-known Routh-Hurwitz conditions in all painstaking detail (the more so as the algebraic manipulations are not always routine). Most of the literature only draws a clear picture when the Routh-Hurwitz conditions imply stability; while the instability results are folklore, we could not find a reference to our satisfaction and reprove them in the appendix. As a twist (for three dimensions), we give a formulation that directly uses the matrix and bypasses the coefficients of the characteristic polynomials.

\section{The model}

To clarify some general issues, we first present a model with one host and an unlimited but finite number of different parasites or different strains of the same parasite.

\subsection{Disease-free dynamics}

Without the disease, the population with density $N(t)$ at time $t$ develops as

$$
N^{\prime}=(\beta(N)-\mu(N)) N
$$

where $\beta(N)$ and $\mu(N)$ are the per capita reproduction and mortality rates (of healthy individuals). 
Assumption 1. $\beta(N)$ is a decreasing positive function of $N \geq 0$, i.e. $0<\beta\left(N_{2}\right) \leq \beta\left(N_{1}\right)$ whenever $N_{2} \geq N_{1} \geq 0$, and $\mu(N)$ is an increasing positive function of $N \geq 0$. Both are continuously differentiable at $N>0$ and $\beta^{\prime}(N)-\mu^{\prime}(N)<0$ for all $N>0 . \beta(N)-\mu(N)$ is positive for $N=0$ and negative for large $N>0$.

It follows from these assumptions that there exists a unique number $K>0$ such that

$$
\beta(K)-\mu(K)=0 .
$$

$K$ is called the carrying capacity of the host population in absence of the disease, because $N(t) \rightarrow$ $K$ as $t \rightarrow \infty$ provided $N(0)>0$.

\subsection{Disease dynamics}

The disease (or diseases in case of several parasites) divides the host population, with density $N(t)$ at time $t$, into different parts:

$$
N=S+I, \quad I=I_{1}+\ldots+I_{n} .
$$

$S$ denotes the density of susceptible, uninfected, hosts, $I$ the total density of infected hosts, while $I_{j}$ denotes the density of hosts infected with strain $j$ (or parasite $j$ ). Notice that we restrict ourselves to the case where there are only susceptible and infective individuals; there is no recovery from the disease, and a latency period is ignored as well. To keep the mathematical complexity down, we assume that there is complete cross-protection between the strains. Our stability results can be expected to hold also if cross-protection is almost complete. Examples of partial cross-protection are reported in the survey article [21] and in [34]. The model reads a follows,

$$
\left\{\begin{array}{l}
N=S+\sum_{j=1}^{n} I_{j}, \\
S^{\prime}=\left(S+\sum_{k=1}^{n} q_{k}\left(1-p_{k}\right) I_{k}\right) \beta(N)-\mu(N) S-\frac{C(N) S}{N} \sum_{k=1}^{n} \sigma_{k} I_{k}, \\
I_{j}^{\prime}=I_{j}\left(\sigma_{j} \frac{C(N) S}{N}+q_{j} p_{j} \beta(N)-\mu(N)-\alpha_{j}\right) .
\end{array}\right.
$$

The infection by strain $j$ is vertically transmitted at the probability $p_{j}, p_{j} \in[0,1]$. In human diseases, $p_{j}$ is the probability that the newborn child of a strain $j$ infectious mother is infectious also. In fungal diseases of plants, $p_{j}$ is the probability at which the seeds of a strain $j$ infected plants carry the fungus as well. Individuals infected by strain $j$ reproduce at the reduced rate $q_{j} \beta(N)$, $q_{j} \in[0,1] . \alpha_{j}$ is the additional per capita rate of dying from the disease.

The strain $j$ incidence (number of new horizontal infections with strain $j$ per unit of time) is given by $\sigma_{j} C(N) S \frac{I_{j}}{N}$.

The parameters $\sigma_{j}$ are compound parameters whose exact interpretation depends on the specific horizontal transmission modes of the strains or parasites. In fungal plant diseases, $\sigma_{j}$ factors in the 


\begin{tabular}{|ll|}
\hline$t$ & time \\
$N$ & total density of hosts \\
$S$ & density of susceptible hosts \\
$I_{j}$ & density of infective hosts that are infected by strain $j$ \\
$f_{j}$ & fraction of infective host that are infected by strain $j$ \\
$\bar{f}$ & fraction of infective hosts \\
\hline
\end{tabular}

Table 1: Model variables

average spore production of a typical plant infected by strain $j$ and the conditional probability that an infection occurs once a spore has landed on a susceptible plant. In sexually transmitted diseases, $\sigma_{j}$ combines the average sexual activity of a typical sexually active person and the conditional probability that a given sexual contact between a susceptible and an infective individual actually leads to an infection. The parameters $\sigma_{j}$ will be of central importance in our analysis, and we call them the horizontal transmission coefficients.

The contact [12, p.90/1] (or response [17]) function $C(N)$ describes how the per capita amount or rate of contacts depend on the host population density $N$. These may be direct contacts as in sexually transmitted diseases or indirect contacts as through spores in fungal plant diseases. Again the precise interpretation depends on the type of disease.

$I_{j} / N$ is the conditional probability that a given contact actually occurs with an infective individual that is infected by strain $j$.

In fungal plant diseases, $C(N)$ is proportional to the probability at which a given spore lands on host plants rather than on the soil (or somewhere else where it is wasted) provided that the host plant density is $N$. At low host plant densities, this probability should be roughly proportional to the plant density which suggests that $C(0)=0$. In sexually transmitted diseases, $C(N)$ is proportional to the number of sexual contacts a typical sexually active person makes in a population with density $N$. Some models assume that $C(N)$ is basically independent of $N$ unless the population density is so low that a deterministic model like ours is not valid anyway. This assumption results in what is sometimes called frequency-dependent (or standard) incidence [24] and is a special case of assuming $C(0)>0$. The study of the evolutionary interaction of horizontal and vertical transmission in [29] assumes density-dependent (or mass-action) incidence where $C(N)$ is proportional to $N$ such that $C(N) / N$ does not depend on host density. Our analysis includes both frequency-dependent and density-dependent incidence and all reasonable interpolations between these two extremes. A collection of contact functions that have been used in the literature can be found in [41, Sec.19.1]; another example, $C(N)=\zeta \ln (a+\nu N)$, has been suggested for insect diseases [7, App.B]. 


\section{Endemic coexistence equilibrium (ECE)}

The ECE is a time-independent solution of (2.3) with all components being positive. Let us assume that the $2^{\text {nd }}$ strain is exclusively vertically transmitted, i.e. $\sigma_{2}=0$. At the ECE,

$$
q_{2} p_{2} \beta\left(N^{*}\right)-\mu\left(N^{*}\right)-\alpha_{2}=0 .
$$

Since $\beta-\mu$ is strictly decreasing, this strain uniquely determines the total population size at such an equilibrium. Generically (i.e. unless there is an exceptional parameter constellation), there is no room for another strain that is exclusively vertically transmitted to coexist at endemic equilibrium. We additionally assume that the first strain is horizontally (and possibly also vertically) transmitted, i.e. $\sigma_{1} \neq 0$. At the ECE,

$$
\frac{C\left(N^{*}\right) S^{*}}{N^{*}} \sigma_{1}+q_{1} p_{1} \beta\left(N^{*}\right)-\mu\left(N^{*}\right)-\alpha_{1}=0 .
$$

Since $N^{*}$ is already uniquely determined by the second strain, the first strain uniquely determines the density of the susceptible individuals. Generically, there is no room for another horizontally transmitted strain to coexist at endemic equilibrium.

So, if one strain is exclusively vertically transmitted, at most one exclusively vertically transmitted strain and one horizontally transmitted strain can coexist with each other and the host at equilibrium.

One can also derive a condition under which one exclusively vertically transmitted strain dynamically excludes any other exclusively vertically transmitted strain. Assume that $\sigma_{2}=0=\sigma_{3}$ and $I_{2}(0)>0$ and $I_{3}(0)>0$. Then $I_{2}(t)>0$ and $I_{3}(t)>0$ for all $t>0$. By the product and power rule and the respective differential equations,

$$
\left(I_{3} I_{2}^{-1}\right)^{\prime}=\left(I_{3} I_{2}^{-1}\right)\left(\left[q_{3} p_{3}-q_{2} p_{2}\right] \beta(N)+\alpha_{2}-\alpha_{3}\right) .
$$

Assume that the second strain is less virulent than the third strain in the sense that

$$
\alpha_{2} \leq \alpha_{3} \text { and } q_{3} p_{3} \leq q_{2} p_{2}
$$

and one of the two inequalities is strict. Then $I_{3}(t) I_{2}^{-1}(t) \rightarrow 0$ as $t \rightarrow \infty$ and, since $I_{2} \leq N$ is bounded [13, Thm.4.2], $I_{3}(t) \rightarrow 0$ as $t \rightarrow \infty$.

\subsection{The special case of one horizontally and one vertically transmitted strain reformulated}

We rewrite the model for one horizontally and one (exclusively) vertically transmitted strain in terms of the total host density,

$$
\begin{aligned}
N^{\prime} & =N(\beta(N)-\mu(N))-\sum_{k=1}^{2} I_{k}\left(\left(1-q_{k}\right) \beta(N)+\alpha_{k}\right), \\
I_{1}^{\prime} & =I_{1}\left(\sigma \frac{C(N)}{N}\left(N-I_{1}-I_{2}\right)+q_{1} p_{1} \beta(N)-\mu(N)-\alpha_{1}\right), \\
I_{2}^{\prime} & =I_{2}\left(q_{2} p_{2} \beta(N)-\mu(N)-\alpha_{2}\right) .
\end{aligned}
$$






Table 2: Parameters

We introduce the fractions of strain $j$ infective individuals,

$$
f_{j}=\frac{I_{j}}{N}
$$

By the quotient rule, $f_{j}^{\prime}=f_{j}\left(\frac{I_{j}^{\prime}}{I_{j}}-\frac{N^{\prime}}{N}\right)$. We rewrite the system in terms of the total host density and the fractions of strain $j$ parasites,

$$
\begin{gathered}
N^{\prime}=N\left(\beta(N)-\mu(N)-\sum_{k=1}^{2} f_{k}\left(\left(1-q_{k}\right) \beta(N)+\alpha_{k}\right)\right), \\
f_{1}^{\prime}=f_{1}\left(\sigma C(N)\left(1-f_{1}-f_{2}\right)-\left(1-q_{1} p_{1}\right) \beta(N)-\alpha_{1}\right. \\
\left.\quad+\sum_{k=1}^{2} f_{k}\left(\left(1-q_{k}\right) \beta(N)+\alpha_{k}\right)\right) \\
f_{2}^{\prime}=f_{2}\left(-\left(1-q_{2} p_{2}\right) \beta(N)-\alpha_{2}\right. \\
\left.\quad+\sum_{k=1}^{2} f_{k}\left(\left(1-q_{k}\right) \beta(N)+\alpha_{k}\right)\right) .
\end{gathered}
$$

We make the following overall assumptions for our model.

Assumption 2. All parameters are non-negative. Both strains do some harm to the host, $\alpha_{j}>0$ or $q_{j}<1$ for $j=1,2$. However, neither strain sterilizes the host, i.e. $q_{j}>0$ for $j=1,2$. Furthermore, vertical transmission is imperfect for both strains, $p_{j}<1$ for $j=1,2$.

$C(N)$ is an increasing function of $N \geq 0, C(N)>0$ for $N>0 . C(N)$ is continuously differentiable at $N>0$.

For some intermediate mathematical considerations some of these assumptions will be relaxed, but they are supposed to hold for all final results.

The following is shown in [13]. 
Theorem 3. A (uniquely determined) ECE exists if and only if the following assumptions are satisfied:

(a) $q_{2} p_{2} \beta(0)-\mu(0)-\alpha_{2}>0$.

(b) The vertically transmitted second strain is less harmful than the horizontally transmitted first strain in the following way,

$$
\left(q_{2} p_{2}-q_{1}\right) \beta\left(N^{*}\right)+\alpha_{1}-\alpha_{2}>0,
$$

where $N^{*}$ is the unique solution of

$$
q_{2} p_{2} \beta\left(N^{*}\right)-\mu\left(N^{*}\right)-\alpha_{2}=0 .
$$

(c) The horizontal transmission coefficient is large enough,

$$
\sigma C\left(N^{*}\right)>\frac{\left(q_{2} p_{2}-q_{1} p_{1}\right) \beta\left(N^{*}\right)+\alpha_{1}-\alpha_{2}}{\left(q_{2} p_{2}-q_{1}\right) \beta\left(N^{*}\right)+\alpha_{1}-\alpha_{2}}\left(\left(1-q_{1}\right) \beta\left(N^{*}\right)+\alpha_{1}\right) .
$$

\section{Local asymptotic stability of the ECE}

The stability of the ECE will be analytically studied using the principle of linearized stability and the Routh-Hurwitz criterion. It will turn out that the ECE, as long as it exists, is locally asymptotically stable for density-dependent incidence. It can be unstable for frequency-dependent incidence and extreme parameter values for vertical transmission and fertility reduction. Our analytic results will be supported and extended by numerical simulation of the differential equations and two-dimensional plots of stability boundaries for frequency-dependent incidence.

Remark 4. (a) In the forthcoming illustrations, we choose the following parameters:

$$
\begin{gathered}
p_{1}=0.9, \quad q_{1}=0.02, \quad \alpha_{1}=0.2, \\
p_{2}=0.99, \quad q_{2}=0.9, \quad \alpha_{2}=0.3, \\
\sigma=0.9, \beta(N)=0.5 e^{-0.2 N}, \mu(N)=0.1 .
\end{gathered}
$$

The carrying capacity, see (2.2), is $K \approx 8.05$. The value of $N$ at the ECE, see (3.1), is $N^{*} \approx 0.539$. $K$ and $N^{*}$ are in terms of some unit of population size which is linked to the factor 0.2 in $\beta(N)$.

(b) We will show the dynamics for both frequency-dependent incidence and density-dependent incidence (Figures 4, 5, and 6). For frequency-dependent incidence we use $C(N)=1$, while for density-dependent incidence we use $C(N)=\frac{N}{N^{*}}$, so that the ECE is the same for both incidence types: $f_{1}^{*} \approx 0.393, f_{2}^{*} \approx 0.283, I_{1}^{*} \approx 0.212, I_{2}^{*} \approx 0.152$. The overall fraction of infected hosts $f_{1}^{*}+f_{2}^{*} \approx 0.676$ is not unusually high for infectious diseases with vertical transmission [38].

(c) We will also show two-dimensional plots of stability boundaries for the ECE for frequencydependent incidence (Figures 1, 2, and 3). In these plots, all parameters except the two being varied are as in (a). The dashed lines are the boundaries of the region where the ECE exists. The ECE is unstable within the solid regions. 
We rewrite the formulation (3.6) of the model with one host and two strains in a condensed way,

$$
\begin{aligned}
N^{\prime} & =N\left(\beta(N)-\mu(N)-f_{1} \gamma_{1}(N)-f_{2} \gamma_{2}(N)\right), \\
f_{1}^{\prime} & =f_{1}\left(\left(1-f_{1}-f_{2}\right) \sigma C(N)+f_{1} \gamma_{1}(N)+f_{2} \gamma_{2}(N)-\eta_{1}(N)\right), \\
f_{2}^{\prime} & =f_{2}\left(f_{1} \gamma_{1}(N)+f_{2} \gamma_{2}(N)-\eta_{2}(N)\right),
\end{aligned}
$$

where

$$
\left.\begin{array}{rl}
\gamma_{j}(N) & =\left(1-q_{j}\right) \beta(N)+\alpha_{j} \\
\eta_{j}(N) & =\gamma_{j}(N)+q_{j}\left(1-p_{j}\right) \beta(N) \\
& =\left(1-q_{j} p_{j}\right) \beta(N)+\alpha_{j}
\end{array}\right\} \quad j=1,2 .
$$

We subtract the two equations,

$$
\eta_{j}(N)-\gamma_{j}(N)=q_{j}\left(1-p_{j}\right) \beta(N) .
$$

\subsection{Equilibrium relations}

Since the ECE is not explicitly given except for its population size component, we will use the relations that describe it implicitly.

Assume that the $\operatorname{ECE}\left(N^{*}, f_{1}^{*}, f_{2}^{*}\right) \in(0, \infty)^{3}$ exists. Write

$$
\begin{aligned}
& \beta=\beta\left(N^{*}\right), \mu=\mu\left(N^{*}\right), C=C\left(N^{*}\right), \gamma_{j}=\gamma_{j}\left(N^{*}\right), \eta_{j}=\eta_{j}\left(N^{*}\right), \\
& \beta^{\prime}=\beta^{\prime}\left(N^{*}\right), \mu^{\prime}=\mu^{\prime}\left(N^{*}\right), C^{\prime}=C^{\prime}\left(N^{*}\right), \gamma_{j}^{\prime}=\gamma_{j}^{\prime}\left(N^{*}\right), \eta_{j}^{\prime}=\eta_{j}^{\prime}\left(N^{*}\right) .
\end{aligned}
$$

In the following, we drop * ¿ ¿From Theorem 3 (b),

$$
\gamma_{1}-\eta_{2}=\left(q_{2} p_{2}-q_{1}\right) \beta+\alpha_{1}-\alpha_{2}>0,
$$

and

$$
q_{2} p_{2} \beta-\mu-\alpha_{2}=0 .
$$

This implies $p_{2}>0$ and $\beta>0$. Combined with Assumption 2, $p_{1} \in[0,1), p_{2} \in(0,1), q_{1}, q_{2} \in$ $(0,1]$, further $\alpha_{1}>0$ or $q_{1}<1$, and also $\alpha_{2}>0$ or $q_{2}<1$. By (4.5) and (4.6),

$$
\eta_{1}>\gamma_{1}>\eta_{2}>\gamma_{2}>0
$$

Remark 5. For some intermediate mathematical considerations we will assume that $q_{j}\left(1-p_{j}\right)=0$ for $j=1,2$. In that case we have

$$
\eta_{1}=\gamma_{1}>\eta_{2}=\gamma_{2}
$$


¿From Theorem 3 (c),

$$
\begin{aligned}
\sigma C & >\frac{\left(q_{2} p_{2}-q_{1} p_{1}\right) \beta+\alpha_{1}-\alpha_{2}}{\left(q_{2} p_{2}-q_{1}\right) \beta+\alpha_{1}-\alpha_{2}}\left(\left(1-q_{1}\right) \beta+\alpha_{1}\right) \\
& =\frac{\eta_{1}-\eta_{2}}{\gamma_{1}-\eta_{2}} \gamma_{1}>\gamma_{1} .
\end{aligned}
$$

¿From the equations for $f_{1}$ and $f_{2}$ in (4.3),

$$
\begin{aligned}
-\left(\sigma C-\gamma_{1}\right) f_{1}-\left(\sigma C-\gamma_{2}\right) f_{2} & =-\left(\sigma C-\eta_{1}\right), \\
\gamma_{1} f_{1}+\gamma_{2} f_{2} & =\eta_{2} .
\end{aligned}
$$

¿From the equation for $N$, we have

$$
\beta-\mu=f_{1} \gamma_{1}+f_{2} \gamma_{2}=\eta_{2}
$$

which, by (4.4), is the same as (4.7). We use the second equation of (4.11) in the first to find the fraction of infective individuals

$$
\bar{f}=f_{1}+f_{2}
$$

and then solve for $f_{1}$ and $f_{2}$,

$$
\begin{aligned}
\bar{f} & =\frac{\sigma C-\left(\eta_{1}-\eta_{2}\right)}{\sigma C}, \\
f_{1} & =\frac{\sigma C\left(\eta_{2}-\gamma_{2}\right)+\gamma_{2}\left(\eta_{1}-\eta_{2}\right)}{\sigma C\left(\gamma_{1}-\gamma_{2}\right)} \stackrel{(4.9)}{>} 0, \\
f_{2} & =\frac{\sigma C\left(\gamma_{1}-\eta_{2}\right)-\gamma_{1}\left(\eta_{1}-\eta_{2}\right)}{\sigma C\left(\gamma_{1}-\gamma_{2}\right)} \stackrel{(4.10)}{>} 0, \\
1-\bar{f} & =\frac{\eta_{1}-\eta_{2}}{\sigma C} \stackrel{(4.9)}{>} 0 .
\end{aligned}
$$

Since $\sigma C>\frac{\eta_{1}-\eta_{2}}{\gamma_{1}-\eta_{2}} \gamma_{1}$ by (4.10),

$$
\begin{aligned}
f_{1} \sigma C & =\frac{\sigma C\left(\eta_{2}-\gamma_{2}\right)+\gamma_{2}\left(\eta_{1}-\eta_{2}\right)}{\gamma_{1}-\gamma_{2}} \\
& >\frac{\frac{\eta_{1}-\eta_{2}}{\gamma_{1}-\eta_{2}} \gamma_{1}\left(\eta_{2}-\gamma_{2}\right)+\gamma_{2}\left(\eta_{1}-\eta_{2}\right)}{\gamma_{1}-\gamma_{2}} \\
& =\frac{\eta_{1}-\eta_{2}}{\gamma_{1}-\gamma_{2}} \frac{\gamma_{1}\left(\eta_{2}-\gamma_{2}\right)+\gamma_{2}\left(\gamma_{1}-\eta_{2}\right)}{\gamma_{1}-\eta_{2}} \\
& =\frac{\eta_{1}-\eta_{2}}{\gamma_{1}-\gamma_{2}} \frac{\gamma_{1} \eta_{2}-\gamma_{2} \eta_{2}}{\gamma_{1}-\eta_{2}}=\frac{\eta_{1}-\eta_{2}}{\gamma_{1}-\eta_{2}} \eta_{2} .
\end{aligned}
$$

Hence

$$
f_{1} \sigma C-\eta_{2}>\frac{\eta_{1}-\gamma_{1}}{\gamma_{1}-\eta_{2}} \eta_{2} \stackrel{(4.8)}{>} 0
$$

Remark 6. If $q_{j}\left(1-p_{j}\right)=0$ for $j=1,2$, we have $f_{1} \sigma C-\eta_{2}=0$. See Remark 5 . 


\subsection{The Jacobian matrix}

In the following we will use the principle of linearized stability. In the appendix we have tailored it to the case of a three-dimensional system (Theorem 12). Define

$$
F=1-\bar{f}+q_{1} f_{1}+q_{2} f_{2} \stackrel{(4.13)}{>} 0 .
$$

The Jacobian matrix of the vector field in (4.3) at the coexistence equilibrium $\left(N, f_{1}, f_{2}\right)$ is

$$
J=\left(\begin{array}{ccc}
N b_{1} & -N \gamma_{1} & -N \gamma_{2} \\
f_{1} b_{2} & f_{1}\left(-\sigma C+\gamma_{1}\right) & f_{1}\left(-\sigma C+\gamma_{2}\right) \\
f_{2} b_{3} & f_{2} \gamma_{1} & f_{2} \gamma_{2}
\end{array}\right)
$$

where

$$
\begin{aligned}
& b_{1}=\beta^{\prime}-\mu^{\prime}-f_{1} \gamma_{1}^{\prime}-f_{2} \gamma_{2}^{\prime}, \\
& b_{2}=\left(1-f_{1}-f_{2}\right) \sigma C^{\prime}+f_{1} \gamma_{1}^{\prime}+f_{2} \gamma_{2}^{\prime}-\eta_{1}^{\prime}, \\
& b_{3}=f_{1} \gamma_{1}^{\prime}+f_{2} \gamma_{2}^{\prime}-\eta_{2}^{\prime} .
\end{aligned}
$$

For later use, we record the following equations which follow from (4.4), (4.12), and (4.15),

$$
\begin{aligned}
b_{1} & =\left(1-\bar{f}+q_{1} f_{1}+q_{2} f_{2}\right) \beta^{\prime}-\mu^{\prime} \\
& =F \beta^{\prime}-\mu^{\prime}<0, \\
b_{1}+b_{2} & =q_{1} p_{2} \beta^{\prime}-\mu^{\prime}+(1-\bar{f}) \sigma C^{\prime}, \\
b_{1}+b_{3} & =q_{2} p_{2} \beta^{\prime}-\mu^{\prime} .
\end{aligned}
$$

Though $J$ is the Jacobian matrix, we will study $J$ as a matrix in its own right and, in this section, only assume the following inequalities instead of (4.8),

$$
\eta_{1} \geq \gamma_{1}>\eta_{2} \geq \gamma_{2}
$$

See (4.8) and Remark 5. We have

$$
\begin{aligned}
& \operatorname{det} J=N f_{1} f_{2}\left|\begin{array}{ccc}
b_{1} & -\gamma_{1} & -\gamma_{2} \\
b_{2} & -\sigma C+\gamma_{1} & -\sigma C+\gamma_{2} \\
b_{3} & \gamma_{1} & \gamma_{2}
\end{array}\right| \\
& =N f_{1} f_{2}\left|\begin{array}{ccc}
b_{1} & -\gamma_{1} & -\gamma_{2} \\
b_{2}+b_{1} & -\sigma C & -\sigma C \\
b_{3}+b_{1} & 0 & 0
\end{array}\right|=N f_{1} f_{2}\left(b_{3}+b_{1}\right) \sigma C\left(\gamma_{1}-\gamma_{2}\right)
\end{aligned}
$$

By (4.18) and (4.4),

$$
\operatorname{det} J=N f_{1} f_{2}\left(q_{2} p_{2} \beta^{\prime}-\mu^{\prime}\right) \sigma C\left(\gamma_{1}-\gamma_{2}\right)<0
$$

Further

$$
\operatorname{tr} J=N b_{1}+f_{1}\left(-\sigma C+\gamma_{1}\right)+f_{2} \gamma_{2}=N b_{1}-f_{1} \sigma C+f_{1} \gamma_{1}+f_{2} \gamma_{2}
$$


By (4.18) and (4.11),

$$
\operatorname{tr} J=N\left(F \beta^{\prime}-\mu^{\prime}\right)-f_{1} \sigma C+\eta_{2} \stackrel{(4.14)}{<} 0 .
$$

Recall that $J_{k}$ is the $2 \times 2$ matrix that results from $J$ by deleting the $k^{\text {th }}$ column and row,

$$
J_{1}=f_{1} f_{2}\left|\begin{array}{cc}
-\sigma C+\gamma_{1} & -\sigma C+\gamma_{2} \\
\gamma_{1} & \gamma_{2}
\end{array}\right|=f_{1} f_{2} \sigma C\left(\gamma_{1}-\gamma_{2}\right) .
$$

By (4.18),

$$
J_{2}=N f_{2}\left|\begin{array}{cc}
b_{1} & -\gamma_{2} \\
b_{3} & \gamma_{2}
\end{array}\right|=N f_{2}\left(b_{1}+b_{3}\right) \gamma_{2}=N f_{2}\left(q_{2} p_{2} \beta^{\prime}-\mu^{\prime}\right) \gamma_{2}
$$

and

$$
\begin{aligned}
J_{3} & =N f_{1}\left|\begin{array}{cc}
b_{1} & -\gamma_{1} \\
b_{2} & -\sigma C+\gamma_{1}
\end{array}\right|=N f_{1}\left(\left(b_{1}+b_{2}\right) \gamma_{1}-b_{1} \sigma C\right) \\
& =N f_{1}\left(\left(q_{1} p_{1} \beta^{\prime}-\mu^{\prime}+(1-\bar{f}) \sigma C^{\prime}\right) \gamma_{1}-\left(F \beta^{\prime}-\mu^{\prime}\right) \sigma C\right) .
\end{aligned}
$$

We add $J_{2}+J_{3}$ and sort the terms along $\beta^{\prime}, \mu^{\prime}$ and $C^{\prime}$,

$$
\begin{aligned}
J_{2}+J_{3}= & N\left(q_{2} p_{2} f_{2} \gamma_{2}+f_{1}\left(q_{1} p_{1} \gamma_{1}-F \sigma C\right)\right) \beta^{\prime} \\
& +N\left(f_{1}\left(\sigma C-\gamma_{1}\right)-f_{2} \gamma_{2}\right) \mu^{\prime}+N f_{1}(1-\bar{f}) \sigma C^{\prime} \gamma_{1} .
\end{aligned}
$$

We reorganize the terms and use (4.11),

$$
\begin{aligned}
J_{2}+J_{3}= & N\left(q_{1} p_{1} f_{1} \gamma_{1}+q_{2} p_{2} f_{2} \gamma_{2}-F f_{1} \sigma C\right) \beta^{\prime} \\
& +N\left(f_{1} \sigma C-\eta_{2}\right) \mu^{\prime}+N f_{1}(1-\bar{f}) \sigma C^{\prime} \gamma_{1} .
\end{aligned}
$$

We introduce some abbreviations,

$$
J_{1}+J_{2}+J_{3}=a_{0}-a_{1} N \beta^{\prime}+a_{2} N \mu^{\prime}+a_{3} N C^{\prime},
$$

with

$$
\begin{array}{ll}
a_{0}=f_{1} f_{2} \sigma C\left(\gamma_{1}-\gamma_{2}\right), & a_{1}=F f_{1} \sigma C-q_{1} p_{1} f_{1} \gamma_{1}-q_{2} p_{2} f_{2} \gamma_{2} \\
a_{2}=f_{1} \sigma C-\eta_{2}, & a_{3}=f_{1}(1-\bar{f}) \sigma \gamma_{1} .
\end{array}
$$

Lemma 7. All $a_{j}, j=0, \ldots, 3$, are positive except that $a_{2}=0$ if and only if $q_{j}\left(1-p_{j}\right)=0$ for $j=1,2$.

Proof. $a_{0}>0$ by (4.8) and $a_{3}>0$ by the fourth line of (4.13). The statements concerning $a_{2}$ follow from (4.14) and Remark 6. As for $a_{1}$, by (4.15),

$$
a_{1}=(1-\bar{f}) f_{1} \sigma C+\left(q_{1} f_{1}+q_{2} f_{2}\right)\left(a_{2}+\eta_{2}\right)-q_{1} p_{1} f_{1} \gamma_{1}-q_{2} p_{2} f_{2} \gamma_{2}
$$


By the fourth line in (4.13),

$$
a_{1}=f_{1}\left(\eta_{1}-\eta_{2}\right)+\left(q_{1} f_{1}+q_{2} f_{2}\right)\left(a_{2}+\eta_{2}\right)-q_{1} p_{1} f_{1} \gamma_{1}-q_{2} p_{2} f_{2} \gamma_{2} .
$$

We reorganize the terms,

$$
a_{1}=\left(q_{1} f_{1}+q_{2} f_{2}\right) a_{2}+f_{1}\left(\eta_{1}-\eta_{2}\left(1-q_{1}\right)-q_{1} p_{1} \gamma_{1}\right)+f_{2} q_{2}\left(\eta_{2}-p_{2} \gamma_{2}\right) .
$$

Since $\eta_{1} \geq \gamma_{1}$

$$
a_{1} \geq f_{1}\left(q_{1} a_{2}+\eta_{1}\left(1-p_{1} q_{1}\right)-\eta_{2}\left(1-q_{1}\right)\right)>0
$$

By (4.22), $J_{1}+J_{2}+J_{3}>0$. We rewrite the determinant (4.20) and the trace (4.21) of $J$ in terms of (4.23),

$$
\operatorname{det} J=N\left(q_{2} p_{2} \beta^{\prime}-\mu^{\prime}\right) a_{0}, \quad \operatorname{tr} J=N\left(F \beta^{\prime}-\mu^{\prime}\right)-a_{2} \text {. }
$$

So the Routh-Hurwitz coefficient of the Jacobian matrix $J$ satisfies

$$
\begin{aligned}
\hbar J:= & \operatorname{det} J-(\operatorname{tr} J)\left(J_{1}+J_{2}+J_{3}\right) \\
= & N\left(q_{2} p_{2} \beta^{\prime}-\mu^{\prime}\right) a_{0} \\
& -\left(N\left(F \beta^{\prime}-\mu^{\prime}\right)-a_{2}\right)\left(a_{0}-a_{1} N \beta^{\prime}+a_{2} N \mu^{\prime}+a_{3} N C^{\prime}\right) \\
= & N\left(q_{2} p_{2} \beta^{\prime}-\mu^{\prime}\right) a_{0} \\
& +\left(N\left(\mu^{\prime}-F \beta^{\prime}\right)+a_{2}\right)\left(a_{0}-a_{1} N \beta^{\prime}+a_{2} N \mu^{\prime}+a_{3} N C^{\prime}\right) .
\end{aligned}
$$

We organize this expression along $\beta^{\prime}, \mu^{\prime}, C^{\prime}$,

$$
\begin{aligned}
\hbar J= & N \beta^{\prime}\left(q_{2} p_{2}-F\right) a_{0}+a_{2} a_{0} \\
& +\left(N\left(\mu^{\prime}-F \beta^{\prime}\right)+a_{2}\right)\left(-a_{1} N \beta^{\prime}+a_{2} N \mu^{\prime}+a_{3} N C^{\prime}\right) .
\end{aligned}
$$

Since $\mu$ is increasing, we obtain the following first stability result from Theorem 12 .

Theorem 8. The ECE is locally asymptotically stable if the per capita birth rate $\beta$ does not depend on the population density $N$ and $p_{j}<1$ and $q_{j}>0$ for $j=1,2$.

Proof. If $\beta$ does not depend on $N, \beta^{\prime}=0$ and, by (4.26) and Lemma 7,

$$
\hbar J=a_{2} a_{0}+\left(N \mu^{\prime}+a_{2}\right)\left(a_{2} N \mu^{\prime}+a_{3} N C^{\prime}\right) \geq a_{2} a_{0}>0 .
$$


We mention that the ECE is globally stable for initial data with $N(0)>0, f_{1}(0)>0$ and $f_{2}(0)>0$ if both $\beta$ and $C$ do not depend on the population density $N$ [9, Cor.6.6]. Since $\mu$ is increasing, we can drop the terms multiplied by $\mu^{\prime}$ in (4.26),

$$
\hbar J \geq N \beta^{\prime}\left(q_{2} p_{2}-F\right) a_{0}+a_{2} a_{0}+\left(a_{2}-F N \beta^{\prime}\right)\left(-a_{1} N \beta^{\prime}+a_{3} N C^{\prime}\right)
$$

with equality holding if $\mu$ is constant. Since all $a_{j} \geq 0$,

$$
\hbar J \geq N \beta^{\prime}\left(q_{2} p_{2}-F\right) a_{0}-N \beta^{\prime} a_{1} a_{2}-F N \beta^{\prime}\left(-a_{1} N \beta^{\prime}+a_{3} N C^{\prime}\right)
$$

with equality holding if $\mu$ and $C$ are constant and $a_{2}=0$.

If $a, b \in \mathbb{R}$, we define

$$
b \succ a \quad \text { and } \quad b \succeq a
$$

if $b>c a$ and $b \geq c a$ respectively for some $c>0$. Notice that both relations are transitive and that $a>0, b \succeq a$ implies $b>0$ as does $a \geq 0, b \succ a$. Further we use $b \bowtie a$ if $b$ and $a$ have the same sign.

Since $\beta^{\prime} \leq 0$, we have the following result from (4.27) and Theorem 12.

Proposition 9. (a) $\hbar J \succeq\left(F-q_{2} p_{2}\right) a_{0}+a_{1} a_{2}+F a_{3} N C^{\prime}$, and the ECE is locally asymptotically stable if the right hand side is positive.

(b) If $\mu$ and $C$ are constant and $q_{j}\left(1-p_{j}\right)=0$ for $j=1,2$, then

$$
\hbar J \bowtie\left(F-q_{2} p_{2}\right) a_{0}+F\left(-a_{1} N \beta^{\prime}\right) .
$$

and the matrix $J$ in (4.16) has one negative eigenvalue and two complex conjugate eigenvalues with positive real part if the right hand side of this sign relation is negative.

Proof. See Theorem 12. If $q_{j}\left(1-p_{j}\right)=0$, then $\eta_{j}=\gamma_{j}$ by (4.5) and $a_{2}=0$ by (4.23) and Remark 6.

We see from this theorem that the ECE is locally asymptotically stable if $F-q_{2} p_{2} \geq 0$ and $a_{2}>0$. Recall (4.15),

$$
F=1-\bar{f}+q_{1} f_{1}+q_{2} f_{2} \geq 1+\bar{f}\left(\min \left\{q_{1}, q_{2}\right\}-1\right) \geq \min \left\{q_{1}, q_{2}\right\} .
$$

Hence $F-q_{2} p_{2} \geq \min \left\{q_{1}-q_{2} p_{2}, q_{2}\left(1-p_{2}\right)\right\}$. Since $a_{2}>0$ if $q_{j}>0$ and $p_{j}<1$ for $j=1$, we have the following result.

Theorem 10. The ECE is locally asymptotically stable if $q_{j}>0$ and $p_{j}<1$ for $j=1,2$ and $q_{1} \geq q_{2} p_{2}$

Condition (b) in Theorem 3 which is necessary for the existence of the ECE then requires that the difference between the disease death rates $\alpha_{1}-\alpha_{2}$ is sufficiently large. In particular, the ECE is locally asymptotically stable if the HT strain does not reduce the fertility of the host. 

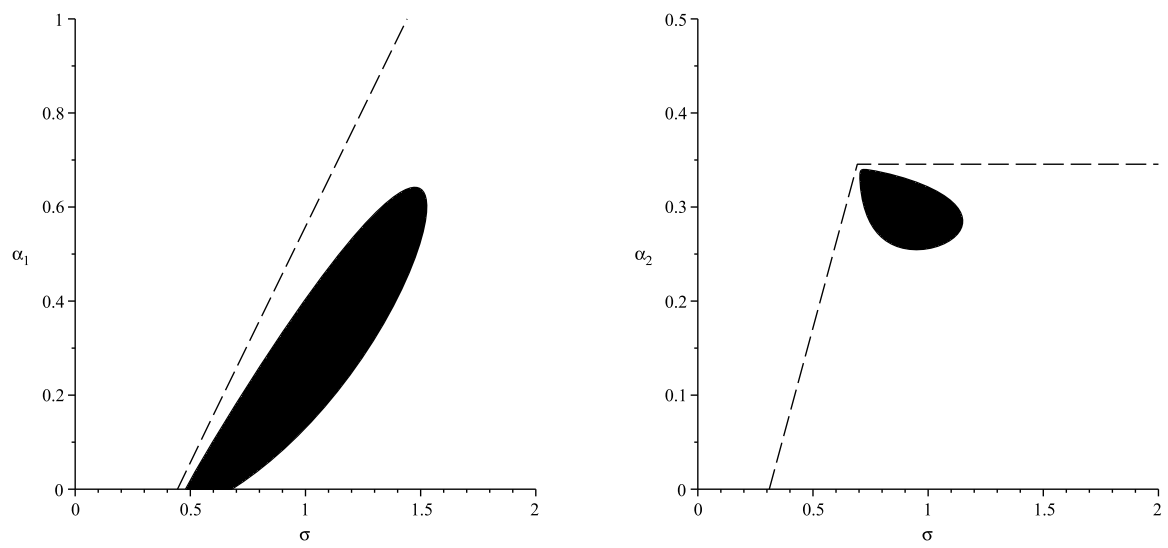

Figure 1: Curves of stability change for frequency-dependent incidence, left $\left(\sigma, \alpha_{1}\right)$, right $\left(\sigma, \alpha_{2}\right)$. See Remark 4 (c).

\section{Stability if the coefficient of horizontal transmission is either slightly super-threshold or large}

By Theorem 3, the ECE exists when the coefficient of horizontal transmission, $\sigma$, is larger than some threshold value $\sigma^{\sharp}$. Further the population size $N$ at the ECE does not depend on $\sigma>\sigma^{\sharp}$. By (4.10),

$$
\sigma^{\sharp} C=\frac{\eta_{1}-\eta_{2}}{\gamma_{1}-\eta_{2}} \gamma_{1} .
$$

In the following we let $\sigma \searrow \sigma^{\sharp}$. By (4.13), $f_{2} \rightarrow 0$ while $f_{1}$ remains bounded away from 0 . By (4.23), $a_{0} \rightarrow 0$ while $a_{1}, a_{2}, a_{3}$ remain bounded away from 0 . By (4.15), $F \rightarrow 1-\left(1-q_{1}\right) f_{1}$ also remains bounded away from 0 . By Proposition 9 (a), $\hbar J$ remains bounded away from 0 with positive value as $\sigma \searrow \sigma^{\sharp}$, and the ECE is locally asymptotically stable if $\sigma$ is slightly larger than the existence threshold $\sigma^{\sharp}$.

We now turn to large values of $\sigma$. By (4.13),

$$
\left.\begin{array}{rl}
f_{1} & \rightarrow \frac{\eta_{2}-\gamma_{2}}{\gamma_{1}-\gamma_{2}}>0 \\
f_{2} & \rightarrow \frac{\gamma_{1}-\eta_{2}}{\gamma_{1}-\gamma_{2}}>0 \\
-\bar{f} & \rightarrow 0 .
\end{array}\right\} \sigma \rightarrow \infty .
$$

By (4.15),

$$
F \stackrel{\sigma \rightarrow \infty}{\sim} q_{1} f_{1}+q_{2} f_{2}
$$

By Proposition 9 (a) and (4.23),

$$
\begin{aligned}
& \hbar J \succ\left(F-q_{2} p_{2}\right) a_{0}+a_{1} a_{2} \\
& \stackrel{\sigma \rightarrow \infty}{\sim}\left(\left(q_{1} f_{1}+q_{2} f_{2}-q_{2} p_{2}\right) f_{2}\left(\gamma_{1}-\gamma_{2}\right)+\left(q_{1} f_{1}+q_{2} f_{2}\right) f_{1} \sigma C\right) f_{1} \sigma C .
\end{aligned}
$$


The right hand side of (5.3) converges to $\infty$ as $\sigma \rightarrow \infty$, and and the ECE is locally asymptotically stable for sufficiently large $\sigma$ by Proposition 9 (a).

If the horizontal incidence is frequency-dependent, the ECE can loose its stability for intermediate values of $\sigma$. See Figure 1. The stability boundaries have been found numerically by solving the equation $\hbar J=0$ for $\sigma$ and $\alpha_{1}$ (or $\alpha_{2}$ ). These numerical calculations also show that, under frequency-dependent incidence, the stability loss can occur for intermediate values of the disease-death rates $\alpha_{1}$ and $\alpha_{2}$. We have no analytic corroboration of this phenomenon because the dependence of the coefficients $a_{j}$ in (4.23) on $\alpha_{1}$ and $\alpha_{2}$ is quite complex.

\section{Stability for density-dependent incidence}

We assume that the incidence is of mass-action type, in other words density-dependent: $C(N)$ is proportional to $N$. Then $N C^{\prime}=C$ and, by Proposition 9 (a),

$$
\hbar J \succ\left(F-q_{2} p_{2}\right) a_{0}+a_{1} a_{2}+F a_{3} C,
$$

and it is sufficient for the stability of the ECE that the right hand side of this relation is positive. The rest of this section is devoted to showing the latter.

Recall that, for $a, b \in \mathbb{R}$, we have defined the relations

$$
b \succ a \quad \text { and } \quad b \succeq a
$$

by $b>c a$ and $b \geq c a$ respectively for some $c>0$. Notice that both relations are transitive. Further $a>0, b \succeq a$ implies $b>0$ as does $a \geq 0, b \succ a$.

Since $0 \leq p_{j} \leq 1$ and $a_{j} \geq 0$,

$$
\hbar J \succ\left(F-q_{2}\right) a_{0}+a_{1} a_{2}+F a_{3} C,
$$

and, by (4.24),

$$
a_{1} \geq f_{1}\left(\eta_{1}-\eta_{2}\left(1-q_{1}\right)-q_{1} \gamma_{1}\right)
$$

We substitute the second relation into the first, use (4.23), and divide by $f_{1}$,

$$
\begin{aligned}
\hbar J \succeq & \left(F-q_{2}\right) f_{2} \sigma C\left(\gamma_{1}-\gamma_{2}\right) \\
& +\left(f_{1} \sigma C-\eta_{2}\right)\left(\eta_{1}-\eta_{2}\left(1-q_{1}\right)-q_{1} \gamma_{1}\right)+F(1-\bar{f}) \sigma C \gamma_{1} .
\end{aligned}
$$

By the third and fourth equations in (4.13),

$$
\begin{aligned}
\hbar J \succeq & \left(F-q_{2}\right)\left(\sigma C\left(\gamma_{1}-\eta_{2}\right)-\gamma_{1}\left(\eta_{1}-\eta_{2}\right)\right) \\
& +\left(f_{1} \sigma C-\eta_{2}\right)\left(\eta_{1}-\eta_{2}\left(1-q_{1}\right)-q_{1} \gamma_{1}\right)+F\left(\eta_{1}-\eta_{2}\right) \gamma_{1} .
\end{aligned}
$$


Some of the terms with $F$ cancel. Since $\eta_{1} \geq \gamma_{1}$ and $f_{1} \sigma C-\eta_{2}=a_{2} \geq 0$,

$$
\hbar J \succeq\left(F-q_{2}\right) \sigma C\left(\gamma_{1}-\eta_{2}\right)+q_{2} \gamma_{1}\left(\eta_{1}-\eta_{2}\right)+\left(f_{1} \sigma C-\eta_{2}\right)\left(\gamma_{1}-\eta_{2}\right)\left(1-q_{1}\right) .
$$

We collect the terms with $\sigma C$,

$$
\hbar J \succeq\left(F-q_{2}+f_{1}\left(1-q_{1}\right)\right) \sigma C\left(\gamma_{1}-\eta_{2}\right)+q_{2} \gamma_{1}\left(\eta_{1}-\eta_{2}\right)-\eta_{2}\left(\gamma_{1}-\eta_{2}\right)\left(1-q_{1}\right) .
$$

We multiply by $\gamma_{1}-\gamma_{2}$,

$$
\hbar J \succeq a_{6}\left(\gamma_{1}-\eta_{2}\right)+q_{2} \gamma_{1}\left(\eta_{1}-\eta_{2}\right)\left(\gamma_{1}-\gamma_{2}\right)-\eta_{2}\left(\gamma_{1}-\eta_{2}\right)\left(1-q_{1}\right)\left(\gamma_{1}-\gamma_{2}\right)
$$

with

$$
a_{6}=\left(\gamma_{1}-\gamma_{2}\right)\left(F-q_{2}+f_{1}\left(1-q_{1}\right)\right) \sigma C .
$$

Our next steps will yield an inequality for $a_{6}$ which we will then substitute into (6.3). By (4.15),

$$
\begin{aligned}
a_{6} & =\left(\gamma_{1}-\gamma_{2}\right)\left(1-\bar{f}+q_{1} f_{1}+q_{2} f_{2}-q_{2}+f_{1}\left(1-q_{1}\right)\right) \sigma C \\
& =\left(\gamma_{1}-\gamma_{2}\right) \sigma C\left(1-\bar{f}+f_{1}+q_{2} f_{2}-q_{2}\right) .
\end{aligned}
$$

Since $\bar{f}=f_{1}+f_{2}$,

$$
a_{6}=\left(\gamma_{1}-\gamma_{2}\right) \sigma C\left(1-f_{2}\right)\left(1-q_{2}\right)=\left(1-q_{2}\right)\left(\left(\gamma_{1}-\gamma_{2}\right) \sigma C-\left(\gamma_{1}-\gamma_{2}\right) \sigma C f_{2}\right) \text {. }
$$

By the formula for $f_{2}$ in (4.13),

$$
\begin{aligned}
a_{6} & =\left(1-q_{2}\right)\left(\left(\gamma_{1}-\gamma_{2}\right) \sigma C-\sigma C\left(\gamma_{1}-\eta_{2}\right)+\gamma_{1}\left(\eta_{1}-\eta_{2}\right)\right) \\
& =\left(1-q_{2}\right)\left(\sigma C\left(\eta_{2}-\gamma_{2}\right)+\gamma_{1}\left(\eta_{1}-\eta_{2}\right)\right) .
\end{aligned}
$$

By (4.10), $\sigma C>\gamma_{1}$ and

$$
a_{6} \geq \gamma_{1}\left(1-q_{2}\right)\left(\eta_{1}-\gamma_{2}\right) .
$$

We substitute this inequality for $a_{6}$ into (6.3) and drop $q_{1}$,

$$
\begin{aligned}
\hbar J \succeq & \gamma_{1}\left(1-q_{2}\right)\left(\eta_{1}-\gamma_{2}\right)\left(\gamma_{1}-\eta_{2}\right)+q_{2} \gamma_{1}\left(\eta_{1}-\eta_{2}\right)\left(\gamma_{1}-\gamma_{2}\right) \\
& -\eta_{2}\left(\gamma_{1}-\eta_{2}\right)\left(\gamma_{1}-\gamma_{2}\right) .
\end{aligned}
$$

We reorganize the RHS,

$$
\begin{aligned}
\hbar J \succeq & \left(1-q_{2}\right)\left(\gamma_{1}-\eta_{2}\right)\left(\gamma_{1}\left(\eta_{1}-\gamma_{2}\right)-\eta_{2}\left(\gamma_{1}-\gamma_{2}\right)\right) \\
& +q_{2}\left(\gamma_{1}-\gamma_{2}\right)\left(\gamma_{1}\left(\eta_{1}-\eta_{2}\right)-\eta_{2}\left(\gamma_{1}-\eta_{2}\right)\right) .
\end{aligned}
$$

We recall (4.8), $\eta_{1}>\gamma_{1}>\eta_{2}>\gamma_{2}$, and

$$
\begin{aligned}
\hbar J \succeq & \left(1-q_{2}\right)\left(\gamma_{1}-\eta_{2}\right)\left(\gamma_{1}\left(\gamma_{1}-\gamma_{2}\right)-\eta_{2}\left(\gamma_{1}-\gamma_{2}\right)\right) \\
& +q_{2}\left(\gamma_{1}-\gamma_{2}\right)\left(\gamma_{1}\left(\gamma_{1}-\eta_{2}\right)-\eta_{2}\left(\gamma_{1}-\eta_{2}\right)\right) \\
= & \left(\gamma_{1}-\gamma_{2}\right)\left(\gamma_{1}-\eta_{2}\right)^{2}>0 .
\end{aligned}
$$

By Proposition 9 (a), the ECE is locally asymptotically stable. 

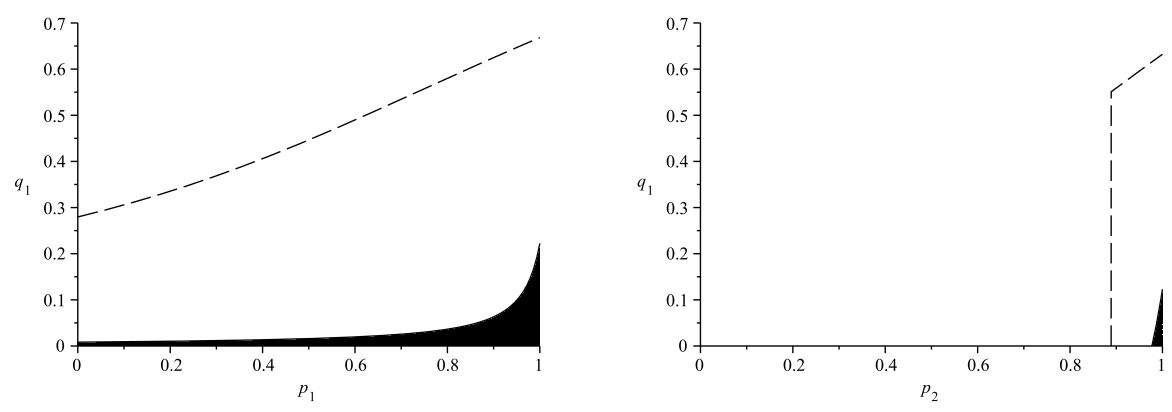

Figure 2: Curves of stability change for frequency-dependent incidence, left $\left(p_{1}, q_{1}\right)$, right $\left(p_{2}, q_{1}\right)$. See Remark 4 (c).

\section{Instability can occur for frequency-dependent incidence}

Assume that $\mu$ is constant. We consider frequency-dependent incidence, i.e., $C$ is constant as well. For a moment, we assume that $p_{2}=1=q_{2}$, and $p_{1}=1$ or $q_{1}=0$. These are boundary cases of the assumptions $q_{j} \in(0,1)$ and $p_{j}<1, j=1,2$, that we have made for the existence of the ECE. We make this assumption, nevertheless, in order to get information on the eigenvalues of the Jacobian matrix $J$. Since these depend continuously on the entries of the matrix, we can then draw conclusions for the case that $p_{2}$ and $q_{2}$ are close to one and that $p_{1}$ is close to one or $q_{1}$ is close to 0 .

We use $\bowtie$ if two real numbers have the same sign. This establishes a transitive relation. By Proposition 9 (b),

$$
\hbar J \bowtie(F-1) a_{0}+F\left(-a_{1} N \beta^{\prime}\right) .
$$

By (4.13) and (4.23),

$$
\begin{aligned}
1-\bar{f} & =\frac{\gamma_{1}-\gamma_{2}}{\sigma C}, \quad f_{1}=\frac{\gamma_{2}}{\sigma C}, \quad f_{2}=1-\frac{\gamma_{1}}{\sigma C}, \\
a_{0} & =\gamma_{2} f_{2}\left(\gamma_{1}-\gamma_{2}\right), \quad a_{1}=f_{1}\left(\gamma_{1}-\gamma_{2}\right)\left(1-q_{1}\right) .
\end{aligned}
$$

Notice that all expressions except $N \beta^{\prime}(N)$ do not depend on $N$ but on $\beta(N)=\mu+\alpha_{2}$ (Theorem 3 (b)). By choosing $\sigma C>0$ large enough and $q_{1}<1$, we make the coexistence equilibrium feasible and $F<1, a_{0}>0$. Let $\psi$ be any strictly decreasing positive function on $\mathbb{R}_{+}$with $\psi(0)>\mu+\alpha_{2}$, $\psi(x) \rightarrow 0$ as $x \rightarrow \infty$. We make the ansatz

$$
\beta(N)=\psi(b+\epsilon N), \quad N \geq 0,
$$

with $b \geq 0$ and $\epsilon>0$. Equation (4.7) for the equilibrium host population size, $\beta(N)=\mu+\alpha_{2}$, then takes the form

$$
b+\epsilon N=\psi^{-1}\left(\mu+\alpha_{2}\right) .
$$



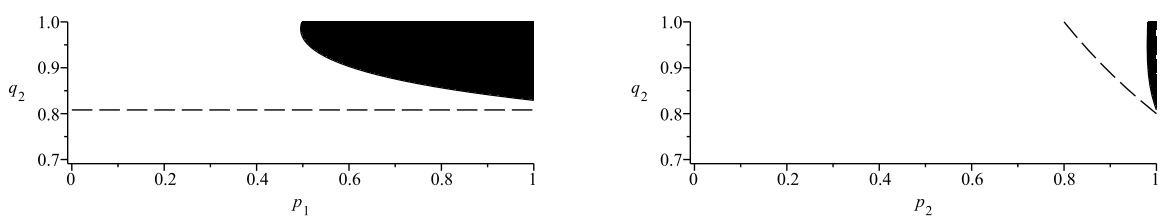

Figure 3: Curves of stability change for frequency-dependent incidence, left $\left(p_{1}, q_{2}\right)$, right $\left(p_{2}, q_{2}\right)$. See Remark 4 (c).

Further

$$
N \beta^{\prime}(N)=\psi^{\prime}(b+\epsilon N) \epsilon N=\psi^{\prime}\left(\psi^{-1}\left(\mu+\alpha_{2}\right)\right) \epsilon N .
$$

We now choose $\epsilon N>0$ so small that $\hbar J<0$ and $b:=\psi^{-1}\left(\mu+\alpha_{2}\right)-\epsilon N \geq 0$. By manipulating $\epsilon$ we can have $N$ as small or as large as we want. This will not change the ratio of $N$ to the carrying capacity $K$ which is

$$
\frac{N}{K}=\frac{\psi^{-1}\left(\mu+\alpha_{2}\right)-b}{\psi^{-1}(\mu)-b}=\frac{\epsilon N}{\psi^{-1}(\mu)-b} .
$$

This shows that the ratio of the host population size at the ECE to the one at the disease-free carrying capacity is small.

By continuity, we can have $\hbar J<0$ also if $p_{2}$ and $q_{2}$ are smaller than 1 , but almost 1 , i.e. the VT strain is almost perfectly vertically transmitted and causes almost no fertility reduction, and if $q_{1}\left(1-p_{1}\right)$ is close enough to 0 , i.e. the HT strain is almost perfectly vertically transmitted or sterilizes the host almost completely. See Figures 2 and 3.

The instability of the ECE follows from Theorem 12. One eigenvalue of the Jacobian matrix is negative, but the other two are complex conjugate with positive real part.

\section{Discussion}

The host-parasite model in Section 3.1 with one exclusively vertically transmitted strain (VT strain for short) and a second horizontally (and perhaps also vertically) transmitted strain (HT strain for short) has many remarkable properties some of which have been presented before. The first is the paradox that the ratio of horizontal to vertical transmission decreases if the coefficient, $\sigma$, of horizontal transmission increases [13]. This phenomenon has also been observed in the simulations of a discrete-time model [31]. The second (also observed in numerical simulations of somewhat different models $[29,31]$ ) is that the vertically transmitted strain that would go extinct by itself can persist by protecting the host against the more virulent horizontally transmitted strain [9].

The third feature, presented in this paper, is the possible loss of stability of the endemic coexistence equilibrium (ECE) for frequency-dependent (alias standard) incidence. This was quite 


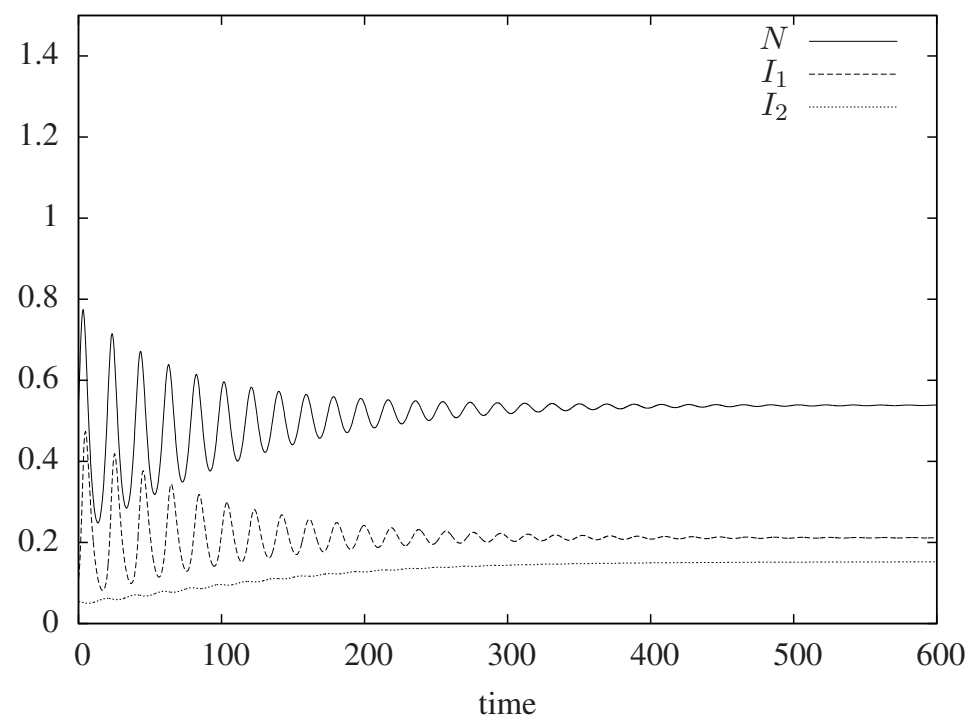

Figure 4: The graphs of $N, I_{1}$, and $I_{2}$ with respect to time, for density-dependent incidence. See Remark 4.

unexpected and was only considered a possibility after, for a long time, finding satisfactory conditions for local stability eluded in the algebraic maze of checking the Routh-Hurwitz criterion.

These are various sufficient conditions that we eventually found for the ECE (provided it exists) to be locally asymptotically stable:

- the per capita host birth rate $\beta$ does not depend on the population density $N$ (Theorem 8 ),

- the HT strain does not reduce the fertility of the host more than the VT strain (but causes more disease deaths) (Theorem 10),

- the coefficient of horizontal transmission $\sigma$ is either very large or slightly above its existence threshold for the ECE (Section 5, Figure 1),

- the incidence is density-dependent (i.e. of mass-action type) (Section 6, Figures 4 and 6).

The ECE is found to be unstable in an oscillatory way (the associated Jacobian matrix has one negative eigenvalue and two complex conjugate eigenvalues with positive real part), if all of the following hold (Section 7):

$\diamond$ frequency-dependent (alias standard) incidence,

and

$\diamond$ the per capita birth rate $\beta$ depends on the population density $N$ (but not too strongly), and the per capita mortality rate $\mu$ only very weakly depends on the population density $N$, and 


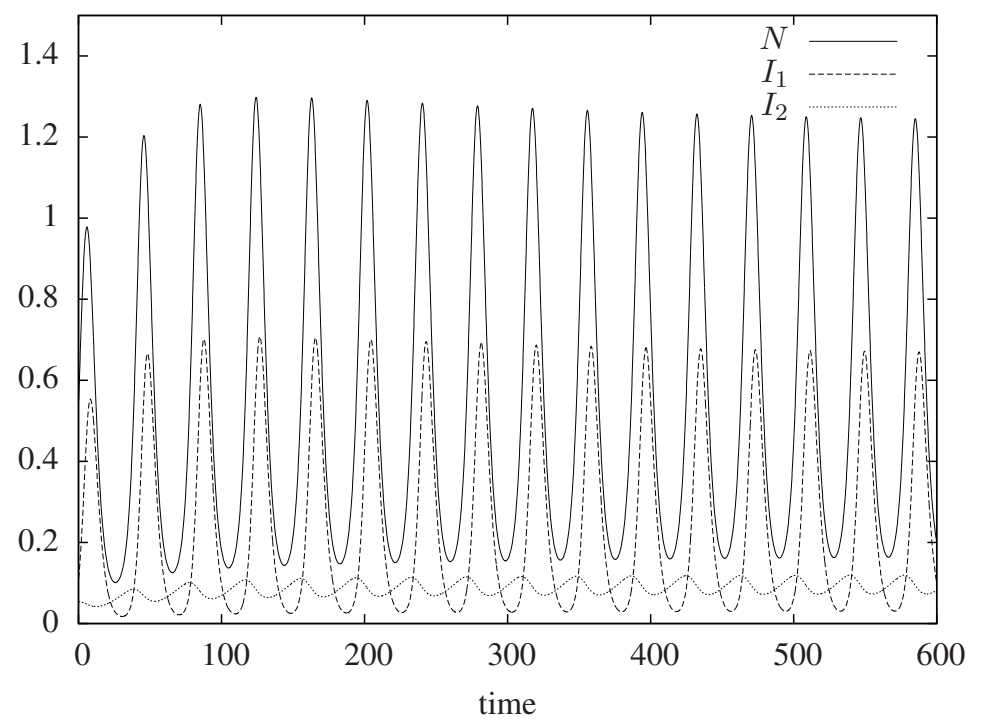

Figure 5: The graphs of $N, I_{1}$, and $I_{2}$ with respect to time, for frequency-dependent incidence. See Remark 4.

$\diamond$ the VT strain is almost perfectly vertically transmitted and causes very little fertility reduction,

and

$\diamond$ the HT strain is almost perfectly vertically transmitted or causes almost complete sterilization.

See Figures 2 and 3. Though we have not proved the occurrence of undamped oscillations analytically, their existence is suggested by the two complex conjugate eigenvalues with positive real part of the Jacobian matrix and has been confirmed by numerical solution of the differential equations (Figures 5 and 6).

Our model has mainly been motivated by endophytic fungal diseases in grasses [13, Sec.7], but applies to many more situations. Among other things, it applies when there are two different parasites rather than two strains of the same parasite that afflict one host (epidemiological interference $[6,11])$.

A model for one host and two viruses with cross-protection is considered in [1, Sec.5]. One virus is exclusively horizontally transmitted, and the other is horizontally and/or vertically transmitted. Frequency-dependent incidence is assumed, and the viruses can cause fatalities, but do not reduce fertility. It is possible to interpret this model in our framework as one with densityindependent per capita birthrate $\beta=b$ and density-dependent natural per capita mortality rate $\mu(N)=b-f(N)$, and vertical transmission rate $p_{2} \beta=b-b_{2}$ where $b, b_{2}$, and $f(N)$ are from the notation used in [1, Sec.5]. So the dynamics in both frameworks agree in so far that the ECE is globally asymptotically stable whenever it exists [1, Thm.1.1(v)(a)][9, Cor.6.6]. If the second 
strain is exclusively vertically transmitted as in our model, the local stability also holds for general (horizontal) incidence of the first strain (Theorem 8).

In comparison, Section 7 confirms the destabilizing potential of disease-reduced host fertility or of other ways the disease affects host reproduction [5, Fig.6] [2, 10, 16, 37, 39].

\subsection{Comparison to other multiple strain models with undamped oscillations}

There is an ongoing interest in which mechanisms can cause undamped oscillations in endemic models $[22,23]$. We have found undamped oscillations in a model of

- SI type, two strains, three ODEs, frequency-dependent (standard) incidence, vertical transmission, fertility reduction, and complete cross-protection.

Most multi-strain models with undamped oscillations that are reported in the literature are considerably more complex and have density-dependent (mass-action) incidence:

- SIR type, three strains, nine ODEs, density-dependent incidence and partial cross-immunity [28],

- SIR type, two strains, age structure (PDE), density-dependent incidence, complete crossprotection and partial cross-immunity $[3,8]$.

A model that has a similarly simple structure as ours is the one in [27] that features

- SIR type, two strains, three ODEs, complete cross-protection and complete cross-immunity, mutation from the first to the second strain ( $\mathrm{R}$ stands for 'removed').

Complete cross-immunity means that an individual that recovers from infection with one strain is immune to all strains. The last model has been formulated for frequency-dependent incidence, but this is not relevant for the large-time behavior because the total population size has a limit as time tends to infinity (nobody dies from the disease and the population turnover is modeled by constant recruitment).

Differently from our model, where the oscillations are driven by some interplay between frequency-dependent incidence and fertility reduction by the disease, the undamped oscillations in [27] appear to be driven by an ongoing mutation from the first to the second strain.

Another simple model two-strain model with undamped oscillations is the one whose dynamics are depicted in [26, Fig.1],

- SIS type with vaccination, two strains with superinfection, three ODEs.

The general model in [26] consists of ordinary and partial differential equations, but [26, Fig.1] considers the special case that the return rate of vaccinated individuals into the susceptible class does not depend on vaccination-age. So the model collapses to a system of four ODEs. Since, differently from our model, the population turnover is of constant recruitment type and there are 


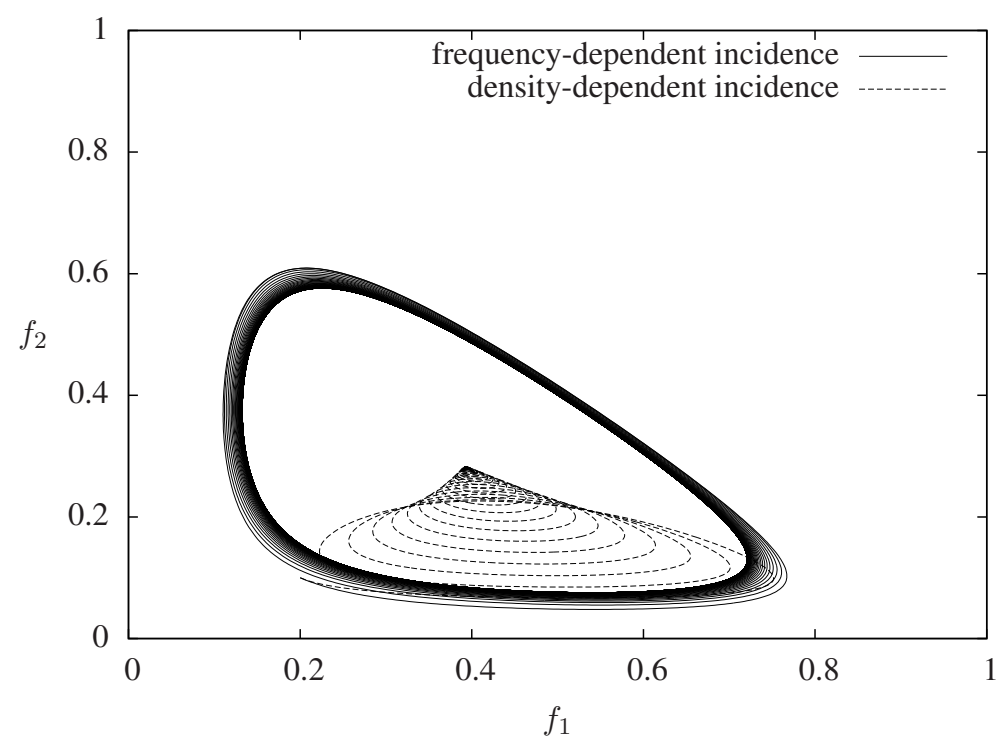

Figure 6: Phase plane projection of $f_{1}$ and $f_{2}$. See Remark 4.

no disease deaths, the total population size converges as time tends to infinity and the system is asymptotically autonomous with a limit system of three ODEs. The model has been formulated for frequency-dependent incidence, but this is not relevant for the large-time behavior because of the convergent total population size. Further it has return of infective and vaccinated individuals into the susceptible class. These last features are missing in an otherwise similar model [32] for which no undamped oscillations are mentioned, but may not have been looked for.

There are other models with multiple parasite strains or multiple parasites that exhibit undamped oscillations, but they contain ingredients like infection-age dependent infectivity or/and return into the susceptible class or isolation that are known to lead to undamped oscillations just by themselves $[33,36]$.

\subsection{Density versus frequency dependence}

In our model, the endemic coexistence equilibrium (ECE) is always locally asymptotically stable for density-dependent (horizontal) incidence, but can be unstable for frequency-dependent incidence (though only for rather extreme parameter values for vertical transmission and fertility reduction). See Section 6 and Section 7 and the Figures 4, 5, and 6. In these figures, the parameters have the same values (see Remark 4). For frequency-dependent incidence we use $C(N)=1$, while for density-dependent incidence we use $C(N)=\frac{N}{N^{*}}$. Here $N^{*}$ is the population size at the ECE and does not depend on the choice of the horizontal incidence. So the ECE is the same for both incidence types. The Routh-Hurwitz coefficient of the Jacobian matrix is $\hbar J \approx-0.000113$ for frequency-dependent incidence, and $\hbar J \approx 0.002308$ for density-dependent incidence, see (4.26).

The same relation between incidence and stability (the ECE is stable for density-dependent 
incidence, but can be unstable for frequency-dependent incidence) has been observed in a model for HIV/AIDS with infection-age dependent infectivity [35, 42].

An opposite relation has been observed for endemic models that include an exposed class and fertility reduction for infectious and possibly exposed individuals: global stability of the endemic equilibrium occurs for frequency-dependent incidence, and undamped oscillations can occur for density-dependent incidence [16, 19, 37]. Similarly, undamped oscillations can occur in SIS endemic models with two hosts and one parasite for density-dependent, but not frequencydependent incidence $[20,24,25]$. Periodic solutions have even been found for two competing host species only one of which is afflicted by a parasite provided that the incidence is density-dependent $[44,45]$. One readily checks that the analogous model in [44] with frequency-dependent incidence has all trajectories converging towards an equilibrium. The mathematical mechanism for the relation between stability and frequency-dependent incidence in these papers is as follows: Rewriting the model in terms of the fraction of infectious (and possible removed) individuals, one can decouple a subsystem of at most two dimensions from the rest of the system and subject it to Poincaré/Bendixson and Dulac theory. If such a reduction of dimensionality does not occur for frequency-dependent incidence, it can as easily or even more easily associate itself with an unstable endemic equilibrium as density-dependent incidence.

\section{A The characteristic polynomial}

Let $A$ be a square matrix of size $n$ and $\mathbb{I}$ the identity matrix of size $n$. Then $\operatorname{det}(A-\lambda \mathbb{I})$ is a polynomial of degree $n$,

$$
\operatorname{det}(A-\lambda \mathbb{I})=\sum_{j=0}^{n} \gamma_{j} \lambda^{j}
$$

as can be shown by induction of the size of the matrix. It is easy to see from row or column expansion that $\gamma_{n}=(-1)^{n}$. Setting $\lambda=0$, we see that $\gamma_{0}=\operatorname{det} A$. In order to determine the other coefficients, remember the following about the differentiation of determinants:

Let $B(\lambda)$ be a family of square matrices of same size, the coefficients of which are differentiable functions of $\lambda$. Since the determinant is a linear function of each of its columns,

$$
\frac{d}{d \lambda} \operatorname{det} B(\lambda)=\sum_{k=1}^{n} \operatorname{det} B_{k}(\lambda)
$$

where $B_{k}(\lambda)$ is the matrix that results from $B(\lambda)$ by replacing the $k^{t h}$ column by its derivative,

$$
B_{k}=\left(\begin{array}{ccccccc}
b_{11} & \ldots & b_{1, k-1} & b_{1 k}^{\prime} & b_{1, k+1} & \ldots & b_{1 n} \\
\vdots & & \vdots & \vdots & \vdots & & \vdots \\
b_{n 1} & \ldots & b_{n, k-1} & b_{n k}^{\prime} & b_{n, k+1} & \ldots & b_{n n}
\end{array}\right)
$$

So

$$
\frac{d}{d \lambda} \operatorname{det}(A-\lambda \mathbb{I})=\sum_{k=1}^{n} \operatorname{det} B_{k}(\lambda)
$$


where $B_{k}(\lambda)$ results from $A-\lambda \mathbb{I}$ by replacing the $k^{\text {th }}$ column by the column which has $(-1)$ in the $k^{\text {th }}$ row and zeros otherwise. Expanding the determinant along this column, $\operatorname{det} B_{k}(\lambda)=$ $-\operatorname{det}\left(\tilde{A}_{k k}-\lambda I\right)$ where $\tilde{A}_{j k}$ is the matrix of size $n-1$ resulting from $A$ by removing the $j^{\text {th }}$ row and the $k^{\text {th }}$ column. Evaluating the derivative at $\lambda=0$ yields

$$
\gamma_{1}=-\sum_{k=1}^{n} A_{k}
$$

where $A_{k}=\operatorname{det} \tilde{A}_{k k}$ is the determinant of the matrix that results from $A$ by deleting the $k^{\text {th }}$ column and row. One can continue this differentiation procedure, but the expressions one obtain become unwieldy most of the time. If one differentiates $n-1$ times and evaluates at $\lambda=0$, one obtains sums of determinants of matrices that result from $A$ by $n-1$ times removing rows and columns of the same number and are matrices of size 1 consisting of a diagonal entry of $A$. In the process one obtains a specific diagonal entry as many times as there are permutations of a set with $n-1$ elements, i.e., $(n-1)$ ! times. This shows that

$$
\gamma_{n-1}=(-1)^{n-1} \sum_{k=1}^{n} a_{k k}=(-1)^{n-1} \operatorname{tr} A
$$

is the trace of $A$. By the fundamental theorem of algebra, with $\lambda_{1}, \ldots, \lambda_{n}$ being the eigenvalues of $A$,

$$
\begin{aligned}
& \operatorname{det}(A-\lambda \mathbb{I})=\prod_{k=1}^{n}\left(\lambda_{k}-\lambda\right) \\
= & (-1)^{n} \lambda^{n}+(-1)^{n-1} \lambda^{n-1} \sum_{k=1}^{n} \lambda_{k}+\cdots-\lambda \sum_{k=1}^{n} \prod_{\substack{j \neq k \\
j=1}}^{n} \lambda_{j}+\prod_{k=1}^{n} \lambda_{k} .
\end{aligned}
$$

A comparison of coefficients shows that

$$
\operatorname{det} A=\prod_{k=1}^{n} \lambda_{k}, \quad \operatorname{tr} A=\sum_{k=1}^{n} \lambda_{k}, \quad \sum_{k=1}^{n} A_{k}=\sum_{k=1}^{n} \prod_{\substack{j \neq k \\ j=1}}^{n} \lambda_{j} .
$$

We summarize these results for quadratic matrices of size 3 ,

$$
\operatorname{det}(A-\lambda \mathbb{I})=-\lambda^{3}+(\operatorname{tr} A) \lambda^{2}-\left(\sum_{k=1}^{3} A_{k}\right) \lambda+\operatorname{det} A
$$

Further we have the following relations for the eigenvalues $\lambda_{1}, \lambda_{2}, \lambda_{3}$ :

$$
\begin{aligned}
\operatorname{det} A & =\lambda_{1} \lambda_{2} \lambda_{3}, \\
\operatorname{tr} A & =\lambda_{1}+\lambda_{2}+\lambda_{3}, \\
A_{1}+A_{2}+A_{3} & =\lambda_{1} \lambda_{2}+\lambda_{1} \lambda_{3}+\lambda_{2} \lambda_{3} .
\end{aligned}
$$


Proposition 11 (Routh-Hurwitz criterion). Consider the polynomial equation $\lambda^{3}+a_{1} \lambda^{2}+a_{2} \lambda+$ $a_{3}=0$. The following hold if $a_{1}>0$ and $a_{3}>0$ :

(a) If $a_{2} a_{1}>a_{3}$, all roots $\lambda$ have strictly negative real parts.

(b) If $a_{2} a_{1}-a_{3}=0$, then one root is strictly negative and two roots are purely imaginary and conjugate to each other.

(c) If $a_{2} \geq 0$ and $a_{2} a_{1}<a_{3}$, then one root is strictly negative and two roots are complex conjugate and have strictly positive real parts.

In particular, all roots have strictly negative real parts if and only if $a_{1}>0, a_{3}>0$, and $a_{1} a_{2}>a_{3}$.

Assume that the coefficients $a_{j}$ of the polynomial are continuously differentiable functions on an open interval I containing 0 and that $a_{j}(0)>0$ for $j=1,3$ and $a_{1}(0) a_{2}(0)=a_{3}(0)$. Then the roots $\lambda_{1}, \lambda_{2}, \lambda_{3}$ are continuously differentiable on some interval $\tilde{I} \subseteq I, 0 \in \tilde{I}, \lambda_{2}(0)$ and $\lambda_{3}(0)$ are imaginary roots conjugate to each other and $\lambda_{2}^{\prime}(0)+\lambda_{3}^{\prime}(0)$ has the same sign as $\left(a_{2} a_{1}-a_{3}\right)^{\prime}(0)$.

Cf. [30, p.490].

Proof. We only prove (a), (b), (c) and the last statement. Let $\lambda_{1}, \lambda_{2}, \lambda_{3}$ be the zeros of the polynomial which, by the fundamental theorem of algebra, equals

$$
\begin{aligned}
& \left(\lambda-\lambda_{1}\right)\left(\lambda-\lambda_{2}\right)\left(\lambda-\lambda_{3}\right) \\
= & \lambda^{3}-\lambda^{2}\left(\lambda_{1}+\lambda_{2}+\lambda_{3}\right)+\lambda\left(\lambda_{1} \lambda_{2}+\lambda_{1} \lambda_{3}+\lambda_{2} \lambda_{3}\right)-\lambda_{1} \lambda_{2} \lambda_{3} .
\end{aligned}
$$

We compare the coefficients,

$$
a_{1}=-\left(\lambda_{1}+\lambda_{2}+\lambda_{3}\right), \quad a_{2}=\lambda_{1} \lambda_{2}+\lambda_{1} \lambda_{3}+\lambda_{2} \lambda_{3}, \quad a_{3}=-\lambda_{1} \lambda_{2} \lambda_{3} .
$$

Notice that also in (a) and (b), $a_{2} \geq 0$. So the following hold:

$$
\lambda_{1}+\lambda_{2}+\lambda_{3}<0, \quad \lambda_{1} \lambda_{2} \lambda_{3}<0, \quad \lambda_{1} \lambda_{2}+\lambda_{1} \lambda_{3}+\lambda_{2} \lambda_{3} \geq 0 .
$$

At least one root is real, let us say $\lambda_{1}$. Suppose that $\lambda_{1} \geq 0$. Then $\lambda_{1}>0$. We multiply the third condition by $\lambda_{1}$,

$$
0 \leq \lambda_{1}^{2}\left(\lambda_{2}+\lambda_{3}\right)+\lambda_{1} \lambda_{2} \lambda_{3}
$$

Since $\lambda_{1} \lambda_{2} \lambda_{3}<0$, we have $\lambda_{2}+\lambda_{3} \geq 0$ and so $\lambda_{1}+\lambda_{2}+\lambda_{3} \geq 0$, a contradiction.

So $\lambda_{1}<0$.

The roots $\lambda_{2}$ and $\lambda_{3}$ are either both real or they are complex conjugates. If $\lambda_{2}$ and $\lambda_{3}$ are both real, we can apply the previous consideration and obtain that all roots are negative.

By (A.7),

$$
\begin{aligned}
a_{3}-a_{1} a_{2} & =\left(\lambda_{1}+\lambda_{2}+\lambda_{3}\right) a_{2}-\lambda_{1} \lambda_{2} \lambda_{3} \\
& =\left(\lambda_{2}+\lambda_{3}\right) a_{2}+\lambda_{1}\left(\lambda_{1} \lambda_{2}+\lambda_{1} \lambda_{3}+\lambda_{2} \lambda_{3}\right)-\lambda_{1} \lambda_{2} \lambda_{3} \\
& =\left(\lambda_{2}+\lambda_{3}\right)\left(a_{2}+\lambda_{1}^{2}\right) .
\end{aligned}
$$


(a) By (A.8), $\lambda_{2}+\lambda_{3}<0$. By our previous considerations, $\lambda_{2}$ and $\lambda_{3}$ are strictly negative or they are complex conjugates. In the second case, their real parts are strictly negative.

(b) We now assume that $a_{2} a_{1}-a_{3}=0$. By (A.8), $\lambda_{2}+\lambda_{3}=0$. Since there is no non-negative root, $\lambda_{2}$ and $\lambda_{2}$ are imaginary and conjugate to each other.

(c) As before $\lambda_{1}<0$ and there are no non-negative roots. By (A.8), $\lambda_{2}+\lambda_{2}>0$ and $\lambda_{2}$ and $\lambda_{3}$ are conjugate complex roots with strictly positive real part.

Since all roots $\lambda_{j}(\xi), j=1,2,3$, are simple in a neighborhood of $\xi=0$, we can apply the implicit functions theorem to the polynomial, and obtain that each $\lambda_{j}(\xi)$ is a continuously differentiable function of $\xi$ in a neighborhood of 0 . By (A.8),

$$
\left(a_{3}-a_{1} a_{2}\right)^{\prime}(0)=\left(\lambda_{2}+\lambda_{3}\right)^{\prime}(0)\left(a_{2}(0)+\lambda_{1}^{2}(0)\right) .
$$

In order to relate Proposition 11 to the eigenvalues of a $3 \times 3$ matrix $A$ we combine (A.6) and (A.7),

$$
a_{1}=-\operatorname{tr} A, \quad a_{2}=\sum_{k=1}^{3} A_{k}, \quad a_{3}=-\operatorname{det} A .
$$

We define the Routh-Hurwitz coefficient of the $3 \times 3$ matrix $A$ as

$$
\hbar A:=\operatorname{det} A-(\operatorname{tr} A)\left(\sum_{k=1}^{3} A_{k}\right)=a_{1} a_{2}-a_{3} .
$$

Here $A_{k}$ is the determinant of the $2 \times 2$ matrix that results from $A$ by deleting the $k^{\text {th }}$ row and column.

Since the eigenvalues of $A$ are the roots of the characteristic polynomial, we obtain the following result by using (A.9) and (A.10) in Proposition 11.

Theorem 12. All eigenvalues of $A$ are strictly negative if and only if the trace and the determinant of $A$ are strictly negative and $\hbar A>0$.

If the trace and the determinant of A are strictly negative, the following hold:

(a) If $\hbar A=0$, then one eigenvalue is strictly negative, $\lambda=\operatorname{tr} A$, and two eigenvalues are purely imaginary and conjugate to each other, $\lambda_{ \pm}= \pm i \omega, \omega^{2}=\frac{\operatorname{det} A}{\operatorname{tr} A}$.

(b) If $\sum_{k=1}^{3} A_{k} \geq 0$ and $\hbar A<0$, then one root is strictly negative and two roots are complex conjugate and have strictly positive real part.

Theorem 13. Consider the parameterized autonomous ODE system

$$
x^{\prime}=g(\theta, x)
$$


with a twice continuously differentiable function $g: I \times V \rightarrow \mathbb{R}^{3}$ where $I$ is an open interval and $V$ an open subset of $\mathbb{R}^{3}$. Assume that there exist equilibria $x^{*}(\theta) \in V, \theta \in I, g\left(\theta, x^{*}(\theta)\right)=0$.

Let $A(\theta)=D_{x} g\left(\theta, x^{*}(\theta)\right)$ be the Jacobian matrix of $g(\theta, \cdot)$ at $x^{*}(\theta)$.

Assume that there is some $\theta_{0} \in I$ such that $\operatorname{tr} A\left(\theta_{0}\right)<0$, $\operatorname{det} A\left(\theta_{0}\right)<0$, and $\hbar A\left(\theta_{0}\right)=0$ and $\frac{d}{d \theta} \hbar A\left(\theta_{0}\right) \neq 0$.

Then there is a Hopf bifurcation at $\theta_{0}$ : there exists an open interval $J \ni 0$ and continuously differentiable functions $\Theta: J \rightarrow I$ and $\omega: J \rightarrow(0, \infty)$ and a family of non-constant periodic solutions $x_{s}, s \in J, x_{s}^{\prime}=g\left(\Theta(s), x_{s}\right)$ such that

(i) $\Theta(0)=\theta_{0}, \quad \omega(0)^{2}=\frac{\operatorname{det} A\left(\theta_{0}\right)}{\operatorname{tr} A\left(\theta_{0}\right)}$.

(ii) $x_{s}$ has period $\omega(s)$.

(iii) $\sup \left|x_{s}(\mathbb{R})\right| \rightarrow 0$ as $s \rightarrow 0$.

\section{Acknowledgements}

We thank two anonymous referees for useful comments. This research was partially supported by NSF grant DMS-0436341 (both authors).

\section{References}

[1] L.J.S. Allen, M. Langlais, C.J. Phillips. The dynamics of two viral infections in a single host population with applications to hantavirus. Math. Biosci., 186 (2003), 191-217.

[2] R.M. Anderson, H.C. Jackson, R.M. May, A.D.M. Smith. Population dynamics of fox rabies in Europe. Nature, 289 (1981), 765-771.

[3] V. Andreasen. Multiple times scales in the dynamics of infectious diseases. Mathematical Approaches to Problems in Resource Management and Epidemiology (C. Castillo-Chavez, S.A. Levin, C.A. Shoemaker, eds.), 142-151, Springer, Berlin Heidelberg, 1989.

[4] V. Andreasen, J. Lin, S.A. Levin. The dynamics of cocirculating influenza strains conferring partial cross-immunity. J. Math. Biol., 35 (1997), 825-842.

[5] C. Banerjee, L.J.S. Allen, J. Salazar-Bravo. Models for an arenavirus infection in a rodent population: consequences of horizontal, vertical and sexual transmission. Math. Biosci. Engin., 5 (2008), 617-645.

[6] F.B. Bang. Epidemiological interference. Intern. J. Epidemiology, 4 (1975), 337-342. 
[7] C.J. Briggs, H.C.J. Godfray. The dynamics of insect-pathogen interactions in stage-structured populations. The American Naturalist, 145 (1995), 855-887.

[8] C. Castillo-Chavez, H.W. Hethcote, V. Andreasen, S.A. Levin, W.M. Liu. Epidemiological models with age structure, proportionate mixing, and cross-immunity. J. Math. Biol., 27 (1989), 233-258.

[9] T. Dhirasakdanon, H.R. Thieme. Persistence of vertically transmitted parasite strains which protect against more virulent horizontally transmitted strains. Modeling and Dynamics of Infectious Diseases (Z. Ma, Y. Zhou, J. Wu, eds.), 187-215, World Scientific, Singapore, 2009.

[10] O. Diekmann, M. Kretzschmar. Patterns in the effects of infectious diseases on population growth. J. Math. Biol., 29 (1991), 539-570.

[11] K. Dietz. Epidemiologic interference of virus populations. J. Math. Biol., 8 (1979), 291-300.

[12] K. Dietz. Overall population patterns in the transmission cycle of infectious disease agents. Population Biology of Infectious Diseases (R.M. Anderson, R.M. May, eds.), 87-102, Springer, Dahlem Konferenzen, Berlin, 1982.

[13] S.H. Faeth, K.P. Hadeler, H.R. Thieme. An apparent paradox of horizontal and vertical disease transmission. J. Biol. Dyn., 1 (2007), 45-62.

[14] Z. Feng, H.R. Thieme. Recurrent outbreaks of childhood diseases revisited: the impact of isolation. Math. Biosci., 128 (1995), 93-130.

[15] Z. Feng, H.R. Thieme. Endemic models with arbitrarily distributed periods of infection. II. Fast disease dynamics and permanent recovery. SIAM J. Appl. Math., 61 (2000), 983-1012.

[16] L.Q. Gao, J. Mena-Lorca, H.W. Hethcote. Variations on a theme of SEI endemic models. Differential Equations and Applications to Biology and Industry (M. Martelli, C.L. Cooke, E. Cumberbatch, B. Tang, H.R. Thieme, eds.), 191-207, World Scientific, Singapore, 1996.

[17] W.M. Getz, J. Pickering. Epidemic models: thresholds and population regulation. The American Naturalist, 121 (1983), 892-898.

[18] D. Greenhalgh. Some results for an SEIR epidemic model with density dependence in the death rate. IMA J. Math. Appl. Med. Biol., 9 (1992), 67-106.

[19] D. Greenhalgh. Hopf bifurcation in epidemic models with a latent period and nonpermanent immunity. Math. Comput. Modelling, 25 (1997), 85-107.

[20] J.V. Greenman, P.J. Hudson. Infected coexistence instability with and without densitydependent regulation. J. Theor. Biol., 185 (1997), 345-356.

[21] E.R. Haine. Symbiont-mediated protection. Proc. R. Soc. B, 275 (2008), 353-361. 
[22] H.W. Hethcote, S.A. Levin. Periodicity in epidemiological models. Applied Mathematical Ecology (S.A. Levin, T.G. Hallam, L.J. Gross, eds.), 193-211, Springer, Berlin Heidelberg, 1989.

[23] H.W. Hethcote, H.W. Stech, P. van den Driessche. Nonlinear oscillations in epidemic models. SIAM J. Appl. Math., 40 (1981), 1-9.

[24] H.W. Hethcote, W. Wang, Y. Li. Species coexistence and periodicity in host-host-pathogen models. J. Math. Biol., 51 (2005), 629-660.

[25] H.W. Hethcote, J. Pickering. Infectious disease and species coexistence: a model of LotkaVolterra form. Am. Nat., 126 (1985), 196-211.

[26] M. Iannelli, M. Martcheva, X.-Z. Li. Strain replacement in an epidemic model with superinfection and perfect vaccination. Math. Biosci., 195 (2005), 23-46.

[27] J. Li, Y. Zhou, Z. Ma, J.M. Hyman. Epidemiological models for mutating pathogens. SIAM J. Appl. Math., 65 (2004), 1-23.

[28] J. Lin, V. Andreasen, S.A. Levin. Dynamics of influenza A drift: the linear three-strain model. Math. Biosci., 162 (1999), 33-51.

[29] M. Lipsitch, S. Siller, M.A. Nowak. The evolution of virulence in pathogens with vertical and horizontal transmission. Evolution, 50 (1996), 1729-1741.

[30] W.-m. Liu. Dose-dependent latent period and periodicity of infectious diseases. J. Math. Biol., 31 (1993), 487-494.

[31] C.M. Lively, K. Clay, M.J. Wade, C. Fuqua. Competitive co-existence of vertically and horizontally transmitted diseases. Evolutionary Ecology Res., 7 (2005), 1183-1190.

[32] M. Martcheva. On the mechanisms with strain replacement in epidemic models with vaccination. Current Developments in Mathematical Biology (R.C. John Boucher, K. Mahdavi, eds.), 149-165, World Scientific, Hackensack, 2007.

[33] M. Martcheva, S.S. Pilyugin. The role of coinfection in multidisease dynamics. SIAM J. Appl. Math., 66 (2006), 843-872.

[34] G. Meijer, A. Leuchtmann. The effects of genetic and environmental factors on disease expression (stroma formation) and plant growth in Brachypodium sylvaticum infected by Epichloë sylvatica. OIKOS, 91 (2000), 446-458.

[35] F.A. Milner, A. Pugliese. Periodic solutions: a robust numerical method for an S-I-R model of epidemics. J. Math. Biol., 39 (1999), 471-492.

[36] M. Nuño, Z. Feng, M. Martcheva, C. Castillo-Chavez. Dynamics of two-strain influenza with isolation and partial cross-immunity. SIAM J. Appl. Math., 65 (2005), 964-982. 
[37] A. Pugliese. An $S \rightarrow E \rightarrow I$ epidemic model with varying population size. Differential Equations Models in Biology, Epidemiology and Ecology (S. Busenberg, M. Martelli, eds.), 121-138, Springer, Berlin Heidelberg, 1991.

[38] K. Saikkonen, S.H. Faeth, M. Helander, T.J. Sullivan. Fungal endophytes: a continuum of interactions with host plants. Annu. Rev. Ecol. Syst., 29 (1998), 319-343.

[39] J.H. Swart. Hopf bifurcation and stable limit cycle behavior in the spread of infectious disease, with special application to fox rabies. Math. Biosci., 95 (1989), 199-207.

[40] H.R. Thieme. Stability change of the endemic equilibrium in age-structured models for the spread of S-I-R type infectious diseases. Differential Equations Models in Biology, Epidemiology and Ecology (S. Busenberg, M. Martelli, eds.), 139-158, Springer, Berlin Heidelberg, 1991.

[41] H.R. Thieme. Mathematics in Population Biology. Princeton University Press, Princeton, 2003.

[42] H.R. Thieme, C. Castillo-Chavez. How may infection-age dependent infectivity affect the dynamics of HIV/AIDS?. SIAM J. Appl. Math., 53 (1993), 1447-1479.

[43] H.R. Thieme, A. Tridane, Y. Kuang. An epidemic model with post-contact prophylaxis of distributed length. II. Stability and oscillations if treatment is fully effective. Math. Model. Nat. Phenom., 3 (2008), 267-293.

[44] P. van den Driessche, M.L. Zeeman. Disease induced oscillations between two competing species. SIAM J. Appl. Dyn. Sys., 3 (2004), 601-619.

[45] E. Venturino. The effects of diseases on competing species. Math. Biosci., 174 (2001), 111131. 